\title{
DIVISION I / COMMISSION 8 / WORKING GROUP DENSIFICATION OF THE OPTICAL REFERENCE FRAME
}

\author{
CHAIR \\ PAST CHAIR \\ MEMBERS
}

\author{
Norbert Zacharias \\ Imants Platais \\ William F. van Altena, Beatrice Bucciarelli, \\ Thomas E. Corbin, Christine Ducourant, \\ Dafydd Wyn Evans, Ralph A. Gaume, \\ Irina I. Kumkova, Brian D. Mason, \\ François Mignard, David G. Monet, \\ Jose L. Muinos Haro, Jean Souchay, \\ Sean E. Urban
}

\section{TRIENNIAL REPORT 2006-2009}

\section{Introduction}

A continuation of this WG was voted for at the IAU GA 2006 in Prague. The International Celestial Reference Frame (ICRF) is defined by the positions of 212 distant quasars at radio wavelengths. The primary, optical reference frame is the Hipparcos Celestial Reference Frame (HCRF), which is the Hipparcos Catalog without astrometric 'problem' stars (in: H. Rickman (ed.) 2001, Proceedings IAU XXIV General Assembly, Transactions IAU XXIVB (San Francisco: ASP), Resolution B1.2). The Tycho-2 catalog with its 2.5 million brightest stars forms the first step in the densification of the optical reference frame. However, the limiting magnitude of about $V=12$ of the Tycho-2 catalog is not sufficient for most applications in astronomy and the goal of this IAU Working Group is to further extend the grid of highly accurate positions and motions toward more and fainter stars. The web site of this WG is at <ad.usno.navy.mil/dens_wg/>.

\section{Developments within the past triennium}

A catalog of the approximately 113000 known quasars was compiled and published (Souchay et al. 2008, A\&A) with emphasis on astrometry. Cross referencing between radio, optical and IR data made it possible to significantly improve the positions of targets in traditional QSO catalogs and to derive interstellar extinction and absolute luminosities.

The Automatic Transit Circle (ATC) at La Palma, formerly known as CAMC, continues to operate under the new owner, the Institute of Astrophysics of the Canary Islands (IAC), managed by ROA. Declination zones between -15 and -40 are being observed. The San Fernando automated transit circle telescope continues observations from the Southern Hemisphere, and a catalog of stars to 16 th mag in the 0 to -30 declination zone is in preparation (Muinos 2009).

Astrometric and photometric properties of the Guide Star Catalog II version 2.3, listing almost a billion objects to mag 21 in the $J$ photographic band, have been extensively 
tested by the OATo and STScI teams against the SDSS, 2MASS and UCAC2 catalogs for the assessment of random and systematics errors. Their analysis has shown that a magnitude-dependent astrometric error of the order of 0.1 to 0.2 arcseconds is present (Bucciarelli et al. 2008, in IAU Symposium No. 248). A significant improvement in astrometric accuracy is expected when using fainter calibrators (Tang et al. 2008, in IAU Symposium No. 248) such as the imminent UCAC3 catalog. A complete review of the construction methods and properties of GSC 2.3 has been accepted for publication in the Astronomical Journal (Lasker, Lattanzi, McLean, et al. 2008).

The Astronomisches Rechen-Institut published the PPM-Extended (Roeser et al. 2008, A\&A), a compiled catalog of over 18 million stars based on the GSC 1.2 and other available data to arrive at accurate proper motions for galactic dynamics studies.

Second-epoch observations for the Yale/San-Juan Southern Proper Motion (SPM) program have continued. CCD frames are obtained with the double astrograph at Cesco Observatory in El Leoncito, Argentina (Girard et al. 2008, in IAU Symposium No. 248). Sky coverage south of -20 degrees declination is essentially complete as of June 2008, with over 16850 pointings recorded and only 76 remaining. Proper-motion determinations for 6 globular clusters (Casetti-Dinescu et al. 2007, AJ, 134, 195) provided a testbed for the combination of the first-epoch photographic plate material with second-epoch CCD astrometry. Results were excellent and indicate that the proper motion uncertainties are dominated by the first-epoch material. Thus, the original plan to obtain 2 -fold overlap with the CCD survey has been modified. Adopting a single-fold observing strategy has permitted the completion of the survey south of -20 degrees despite poor weather and recent mechanical problems with the astrograph dome. Lower-priority fields north of -20 degrees will continue, as will observations of some scientifically important fields where second-epoch plate material exists but for which the added precision and extended baseline of new CCD observations warrant a new epoch. Funds for these continued CCD observations have been included in a proposal to the National Science Foundation.

Work is well underway on the SPM4 catalog that will cover the sky south of -20 degrees. In addition to the reduction of the CCD frames, the PMM pixel data for all of the first-epoch plates (as well as the fraction of the sky covered by second-epoch plates) are being re-reduced in a collaboration with the U.S. Naval Observatory (USNO). It is anticipated that the SPM4 will be completed in Fall 2008, contain roughly 100 million stars and galaxies and be more than 90 percent complete to $V=17.5$.

The SPM and Northern Proper Motion (NPM) first epoch data are also needed to derive proper motions of the faint stars in the USNO CCD Astrograph Catalog (UCAC) program. All scan data from the NPM and SPM plates were obtained from the Naval Observatory Flagstaff Station (NOFS) and processed through a modified StarScan pipeline (Zacharias et al. 2008, PASP, 120, 644) to arrive at global x,y data.

Extensive code development for the final reduction of UCAC has been completed and all 275,000 CCD frames pixel data were processed at the USNO in spring 2008. The complete all-sky UCAC3 catalog will contain positions and proper motions of about 80 million stars ( 8 to $16 \mathrm{mag}$ ) with improved astrometric and photometric accuracy. Completeness of UCAC3 will also be significantly higher than in UCAC2, including high proper motion stars, and several hundred thousand newly discovered double stars (Zacharias 2008, in IAU Symposium No. 248).

Optical design studies for the USNO Robotic Astrometric Telescope (URAT) were completed and the primary mirror obtained in spring 2008. This telescope features two full-size aperture $(0.85 \mathrm{~m})$ corrector lenses to support the secondary mirror without a 
spider construction. Currently no funds are identified to complete this new telescope, however the focal plane assembly for URAT has been funded. The world's largest monolithic CCD chip with 111 million pixels was manufactured in 2007 and tested at the USNO astrograph (Zacharias et al. 2007, SPIE 6690-08). The URAT focal plane consists of 4 of these detectors. For phase 1 of URAT (nicknamed 'U-mouse') this 4-shooter camera is being mounted on the re-furbished USNO $20 \mathrm{~cm}$ astrograph 'redlens'. First light is expected in March 2009 and a new sky survey will commence from Cerro Tololo. A single exposure covers 27 square degrees of sky and a 20-fold overlap of the entire Southern Hemisphere can be completed by 2011 to solve for positions, proper motions and parallax of stars in the 8 to 18 mag range in a $680-750 \mathrm{~nm}$ bandpass. The resulting star catalog will be on the ICRF, directly linking the Hipparcos stars and optical counterparts of compact extragalactic radio reference frame sources.

Platais and collaborators continued with the Deep Astrometric Standard (DAS) initiative (Platais et al. 2008, Commission 8 report in IAU Transactions XXVIB) to provide absolute positions and motions to 25th magnitude in selected areas.

\section{Closing remarks}

The major near future task will be to merge all relevant, astrometric, observed catalogs. This huge task will involve systematic error analysis and applying corrections to bring the data onto a common system, globally as well as locally. New observational data relevant for the densification of the optical reference frame are expected over the next couple of years from projects like U-mouse (U.S. Naval Observatory), PanSTARRS (Univ. of Hawaii and collaborators) and JMAPS (proposed micro-satellite, U.S. Naval Observatory). Even further into the future, the Space Interferometry Mission (SIM Planetquest) will be able to define positions and proper motions of individual celestial objects on the micro-arcsecond level. The Gaia (ESA) mission with expected catalog release near the year 2020 will define the optical reference frame at unprecedented densities and magnitude limits.

Norbert Zacharias

chair of the Working Group 